\title{
Reconstruction and analysis of regulatory gene networks involving human genes associated with main forms of pathozoospermia
}

\author{
Elena V. Ignatieva \\ The Federal Research Center Institute \\ of Cytology and Genetics, SB RAS \\ Novosibirsk, Russia \\ eignat@bionet.nsc.ru \\ Ludmila V. Osadchuk \\ The Federal Research Center Institute \\ of Cytology and Genetics, SB RAS \\ Novosibirsk, Russia \\ losadch@bionet.nsc.ru
}

\author{
Alexander V. Osadchuk \\ The Federal Research Center Institute \\ of Cytology and Genetics, SB RAS \\ Novosibirsk, Russia \\ osadchuk@bionet.nsc.ru;
}

\author{
Maxim A Kleshev \\ The Federal Research Center Institute \\ of Cytology and Genetics, SB RAS \\ Novosibirsk, Russia \\ max82cl1@bionet.nsc.ru
}

\begin{abstract}
The study of the molecular-genetic mechanisms predisposing to decline in male reproductive potential (spermatogenic failure) is an actual problem of reproductive biology. Most often in laboratory studies evaluating male fertility, a study of the quality of ejaculate is used. Thus, a pathological condition called pathozoospermia can be detected if the quality indicators of the ejaculate are decreased. Pathozoospermia can manifest itself in several distinct forms, may occur in many diseases and can be caused by many factors, including genetic ones. To reveal regulatory interactions between genes associated with pathozoospermia, we reconstructed gene regulatory network involving genes harboring allelic variants associated with pathozoospermia. Regulatory network comprised seven genes encoding transcription factors (TFs) for which a set of target genes were predicted by MoLoTool web-service. We identified three key regulatory transcription factors (WT1, AHR, NR0B1) that have the greatest number of target genes in the network. Genes encoding these factors can be considered as the most promising candidate genes for identifying genetic variants associated with pathozoospermia.
\end{abstract}

Keywords - male infertility, spermatogenesis, pathozoospermia, genetic association, gene network, transcription factor, target gene

\section{INTRODUCTION}

The decline in male reproductive potential, identified in many countries of the world, is an important scientific problem and serious social issue [1,2]. Most often in laboratory studies evaluating male fertility, a study of the quality of ejaculate is used. Thus, a pathological condition called pathozoospermia may be revealed if the quality indicators of the ejaculate (defined as the reference values of WHO [3]) are decreased. The most common forms of pathozoospermia are azoospermia, asthenozoospermia, oligozoospermia, teratozoospermia. Pathozoospermia is a syndrome that can manifest itself in several distinct forms, may occur in many diseases and can be caused by many factors, including genetic ones. To reveal regulatory interactions between genes associated with pathozoospermia, we reconstructed gene regulatory network and searched for its key regulators.

\section{MATERIALS AND METHODS}

The list of 68 human genes associated with specific forms of pathozoospermia was formed as it was described in [4]. To identify genes that encode transcription factors, we used AnimalTFDB [5]. DNA sequences of promoter regions (500 bp and $1000 \mathrm{bp}$ ) were extracted from the GRCh38.p12 assembly for all genes using BioMart tool. Transcription factor binding sites (TFBSs) were predicted using MoLoTool web-service (https://molotool.autosome.ru/). MoLoTool enables to scan DNA sequences for TFBSs with position weight matrices presented in HOCOMOCO model collection [6]. We used significance threshold pajusted $<10-4$, which was recommended by the web-service. Thus, the information about the hypothesized transcriptional regulation of genes associated with pathozoospermia was obtained. Networks were visualized using Cytoscape [7]

\section{RESULTS}

Using AnimalTFDB we identified that 10 out of 68 genes associated with pathoszoospermia encoded transcription factors: WT1, AHR, NR0B1, ETV5, AR, NFE2L2, SOX5, AHRR, YBX2, SOX6. Three of them (AHRR, YBX2, SOX6) had no motif information in the HOCOMOCO model collection [6]. Then we scanned promoter regions (500 bp and $1000 \mathrm{bp}$ ) of all 68 genes with MoLoTool and identified a set of TFBSs for each promoter. Next we reconstructed two transcriptional regulatory networks built on data on binding sites for TFs in upstream (500 bp and $1000 \mathrm{bp}$ ) gene regions. These networks contained 60 nodes, 123 edges (when 500 bp regions were analyzed) and 65 nodes, 178 edges (when 1000 bp regions were analyzed). Numbers of TFBSs of each type and numbers of target genes for each TF were calculated for each network (Table 1). We found that the greatest number (46 for $500 \mathrm{bp}$ analyzed, and 58 for $1000 \mathrm{bp}$ analyzed) of target genes had WT1. The other two top transcription factors (AHR and NR0B1) had less regulated genes (for AHR there were 29 and 38 target genes, and for NR0B1 there were 25 and 33 genes). Our findings are in good agreement with results obtained in mice, indicating that WT1 is critical for spermatogenesis via regulation of sertoli cell polarity via Wnt signaling pathway [8]. Furthermore, we revealed three 
regulatory self-loops involving transcription factors WT1, NR0B1, and AR.

TABLE 1. Ranking transcription factors according to the number of target genes in the networks

\begin{tabular}{|c|c|c|c|c|}
\hline \multirow[b]{3}{*}{$\begin{array}{l}\text { Transcrip- } \\
\text { tion factor }\end{array}$} & \multicolumn{4}{|c|}{ Network based on analysis of } \\
\hline & \multicolumn{2}{|c|}{500 bp promoter regions } & \multicolumn{2}{|c|}{1000 bp promoter regions } \\
\hline & $\begin{array}{l}\text { Number of } \\
\text { binding sites }\end{array}$ & $\begin{array}{c}\text { Number } \\
\text { of target } \\
\text { genes }\end{array}$ & $\begin{array}{l}\text { Number of } \\
\text { binding sites }\end{array}$ & $\begin{array}{c}\text { Number } \\
\text { of target } \\
\text { genes }\end{array}$ \\
\hline WT1 & 344 & 46 & 660 & 58 \\
\hline AHR & 46 & 29 & 76 & 38 \\
\hline NR0B1 & 41 & 25 & 59 & 33 \\
\hline ETV5 & 19 & 11 & 60 & 24 \\
\hline $\mathrm{AR}$ & 9 & 7 & 19 & 14 \\
\hline NFE2L2 & 2 & 2 & 6 & 6 \\
\hline SOX5 & 3 & 3 & 5 & 5 \\
\hline $\begin{array}{c}\text { Total } \\
\text { number }\end{array}$ & 123 & 60 & 178 & 65 \\
\hline
\end{tabular}

\section{CONCLUSION}

Using MoLoTool web-service that scans DNA sequences for transcription factor binding sites we revealed potential target genes for seven TFs and reconstructed transcriptional regulatory networks involving 60 and 65 human genes associated with pathozoospermia. We identified three key regulatory transcription factors that had the greatest number of target genes: WT1, AHR, and NR0B1. We expect that further experiments to test binding ability of the predicted binding sites will verify the interactions between these three TFs and their target genes. We propose to keep in mind genes encoding these transcription factors as most promising candidates for investigating the genetic factors predisposing to pathozoospermia.

\section{ACKNOWLEDGMENT}

This study was supported by the grant from the Russian Science Foundation (project No. 19-15-00075).

\section{REFERENCES}

1] C. Krausz, F. Cioppi, A. Riera-Escamilla, "Testing for genetic contributions to infertility: potential clinical impact", Expert Rev Mol Diagn, Vol. 18(4), pp. 331-346, 2018

[2] C. Krausz, A. Riera-Escamilla, "Genetics of male infertility", Nat Rev Urol, Vol. 15(6), pp. 369-384, 2018.

[3] WHO, "WHO laboratory manual for the examination and processing of human semen", 5-th edition, Cambridge Univer. Press. 2010.

https://apps.who.int/iris/bitstream/handle/10665/44261/978924154778 9_eng.pdf

[4] E.V. Ignatieva, A.V. Osadchuk, M.A. Kleshev, L.V. Osadchuk, "Towards a Comprehensive Catalog of Human Genes Associated with Main Forms of Pathozoospermia and its Functional Annotation" Systems Biology and Biomedicine (SBioMed-2020), 2020

[5] H.M. Zhang et al., "AnimalTFDB 2 0:A resource for expression, prediction and functional study of animal transcription factors", Nucleic Acids Res, Vol. 43, pp. 76-81, 2015.
[6] I.V. Kulakovskiy et al., "HOCOMOCO: Towards a complete collection of transcription factor binding models for human and mouse via largescale ChIP-Seq analysis", Nucleic Acids Res, Vol. 46, pp. D252D259, 2017.

[7] P. Shannon et al., "Cytoscape: a software environment for integrated models of biomolecular interaction networks", Genome Res, Vol. 13(11), pp. 2498-504, 2003.

[8] X.N. Wang et al., "The Wilms tumor gene, Wt1, is critical for mouse spermatogenesis via regulation of sertoli cell polarity and is associated with non-obstructive azoospermia in humans", PLoS Genet, Vol.9(8), p.e1003645, 2013.

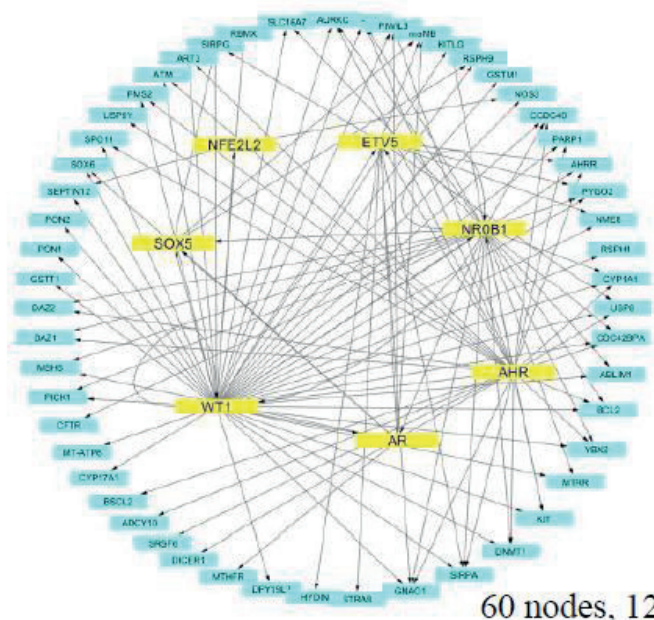

60 nodes, 123 edges

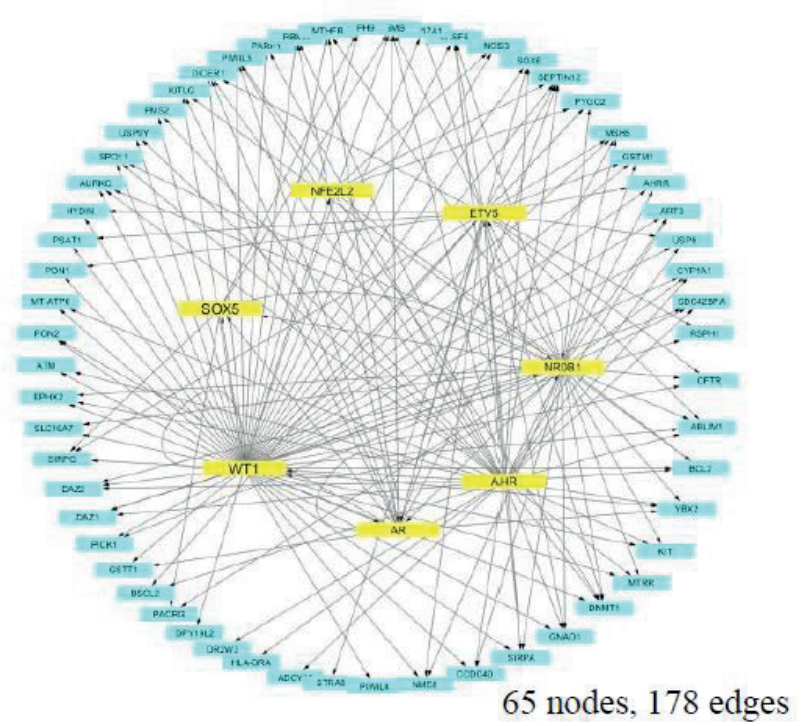

Fig. 1. Gene regulatory networks formed by regulatory interactions between transcription factors and target genes, associated with pathozoospermia. Networks were constructed based on the analysis of 500 $\mathrm{bp}$ (top) and $1000 \mathrm{bp}$ (bottom) promoter regions. Yellow nodes correspond to both genes encoding transcription factors and transcription factors themselfs, blue nodes denote target genes 